\title{
Osservazioni di microvariazioni di raggi cosmici in corrispondenza al passaggio di perturbazioni frontali
}

\author{
M. C. Fazzini, M. Galdi, I. Guidi, P. Randi (*)
}

Ricevuto il 20 Marzo 1967

Rrassunro. - In corrispondenza di perturbazioni atmosferiche frontali a carattere freddo, si osservano, quasi sistematicamente, variazioni ca. ratteristiche della componente totale dei raggi cosmici al suolo, dell'ordine dell'1\% e della durata di alcuni quarti d'ora. Se ne discutono le possibili cause e si conclude, al livello delle conoscenze attuali, circa le possibilità di un effetto di temperatura o di elettricitì atmosferica.

Summarz. - Peculiar small variations of the total component of cosmic rays have been observed during atmospheric cold front perturbations. These variations have an amplitude of the order of $1 \%$ and a duration of a few quarters of an hour. The possible causes are discussed and it is concluded on the possibility of an effect of temperature or of atmospheric electricity.

\section{INTRODUZIONE.}

Si ha ragione di ritenere che perturbazioni meteorologiche di carattere frontale abbiano effetti peculiari e di breve durata (su seala di tempo di quarti d'ora) sulla intensità della radiazione cosmica Secondaria al suolo; detti effetti si aggiungono a quelli derivanti dalle variazioni di pressione o di temperatura, la quale ultima è generalmente misuratia su scala di tempo di parecchie ore. Le cause possibili di tali variazioni che sono state considerate finora sono: l'attività dei campi

(*) Istituto di Fisica Università di Bologna, Gruppo Italiano di Fisica Cosmica; Consiglio Nazionale delle Ricerche 
clettrici atmosferici $\left({ }^{1,2,3,4}\right)$ e le variazioni di temperatura durante il passaggio di fronti $\left({ }^{5,6}\right)$; la loro evidenza è però risultata disomogenea - talvolta contraddittoria.

Con le apparecchiature esistenti presso la stazione, poiché si è in grado di apprezzare variazioni di qualche \%o nell'intervallo di un quarto d'ora, si sono esaminate le valiazioni dei raggi cosmici in corrispondenza al passaggio di perturbazioni frontali.

\section{DESCRIZIONE DEL SISTEMA DI RIVELAZIONE.}

Il sistema di contatori di cui è dotata questa stazione è costituito da quattro rivelatori disposti come mostrato in Fig. 1. Ogni rivelatore ronsiste di uno scintillatore plastico al polistirolo il eui spessore è di circa cm 13 a la cui area è circa $(2 \times 2) \mathrm{m}^{2}$. Le scintillazioni provocate dalle particelle ionizzanti sono viste da fototubi sovrastanti. Oltre ai conteggi singoli, provenienti da ognuno dei quattro rivelatori, vengono registrate le coincidenze tra il contatore A (Fig. 1) ed ognuno dei tre contatori sottostanti $W, B$, E.

Lo spettro differenziale degli impulsi presenta un minimo in corrispondenza di particelle che perdono intorno a $7 \mathrm{MeV}$ (Fig. 2) nello scintillatore $(*)$, quindi il discriminatore, per ragioni di stabilità e di riproducibilitì, viene predisposto in modo da contare tutti gli impulsi di altezza superiore a quella del primo minimo dello spettro. Questo punto corrisponde al minimo della somma dello spettro differenziale dei $\mu\left({ }^{7}, 8\right)$ che è discendente, e di quello della elettrofotonica che è (rescente $\left({ }^{9}\right)$.

I contegur singoli ottenuti da ciascun contatore rappresentano pereiò la radiazione secondaria totale tagliata a 7 MeV. Il totale dei

(*) Questa cifra può essere ragionevolmente giustificata pensando che, in corrispondenza di tale taglio, i neutroni di circa $14 \mathrm{MeV}$ provenienti da un acceleratore Cockroft-Walton posto sotto l'edificio dell'Istituto di Fisica, vengono rivelati o non rivelati a secondo che, in uno stretto intervallo intorno a quella posizione, venga variato in più o in meno la tensione di taglio del discriminatore. Infatti tali neutroni, per arrivare al rivelatore, attraversano circa $50 \mathrm{~cm}$ di materiale murario, cioè perdono in corrispondenza circa il $10 \%$ della loro energia; nello stirolo è contenuto circa il $7,7 \%$ di idrogeno ed il $92,3 \%$ di carbonio; quindi il protone di rinculo, mediante il quale si può rivelare un neutrone nello scintillatore, darà un impulso medio di luce corrispondente ad una particella carica di circa $7 \mathrm{MeV}$. 
conteggi di questo tipo è circa $2.000 .000 / \mathrm{h}$ per ciascun contatore. Il contatore superiore $A$ (Fig. 1) è posto sopra uno strato di $10 \mathrm{~cm}$. di piombo. Si ottengono quindi le coincidenze della geometria cubica

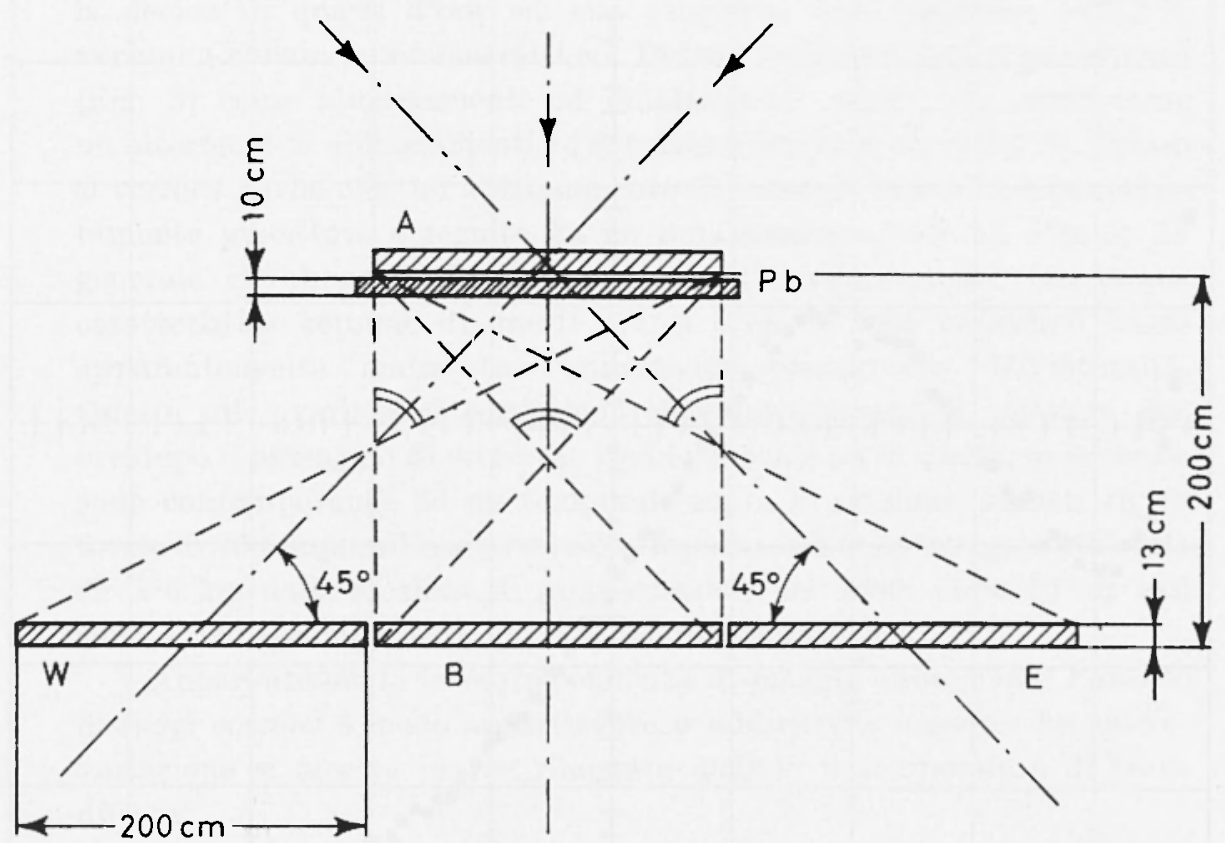

l'ig. 1 - Sezione verticale nella direzione Est-Ovest attraverso il supermonitore di Bologna mostrante la posizione dei pannelli di scintillatore plastico e la traccia dei coni di accettazione.

standard mediante le coincidenze A - B (500.000 conteggi all'om), e quelle inclinate mediamente di $45^{\circ}$ ad Ovest (coincidenze A-E, 150.000 conteggi all'ora) e di $45^{\circ}$ ad Est (coincidenze $A-W, 150.000$ conteggi all'ora).

Dalla Fig. 1 si puó notare che una frazione della parte molle della totale viene schermata dal contatore sovrastante $A$; a calcoli fatti risulta però che tale frazione certamente non supera il 3,5\% della totale. I conteggi ottenuti dai contatori $\mathrm{W}, \Lambda, \mathrm{E}$ vengono corretti per l'effetto di pressione che è il $2,04 \% / \mathrm{oo} / \mathrm{mb}$; essi vengono poi opportunamente sommati e mediati, ottenendo cosi valori finali su scala di tempo di 15 minuti, e con una deviazione standard di circa l'1\%. 


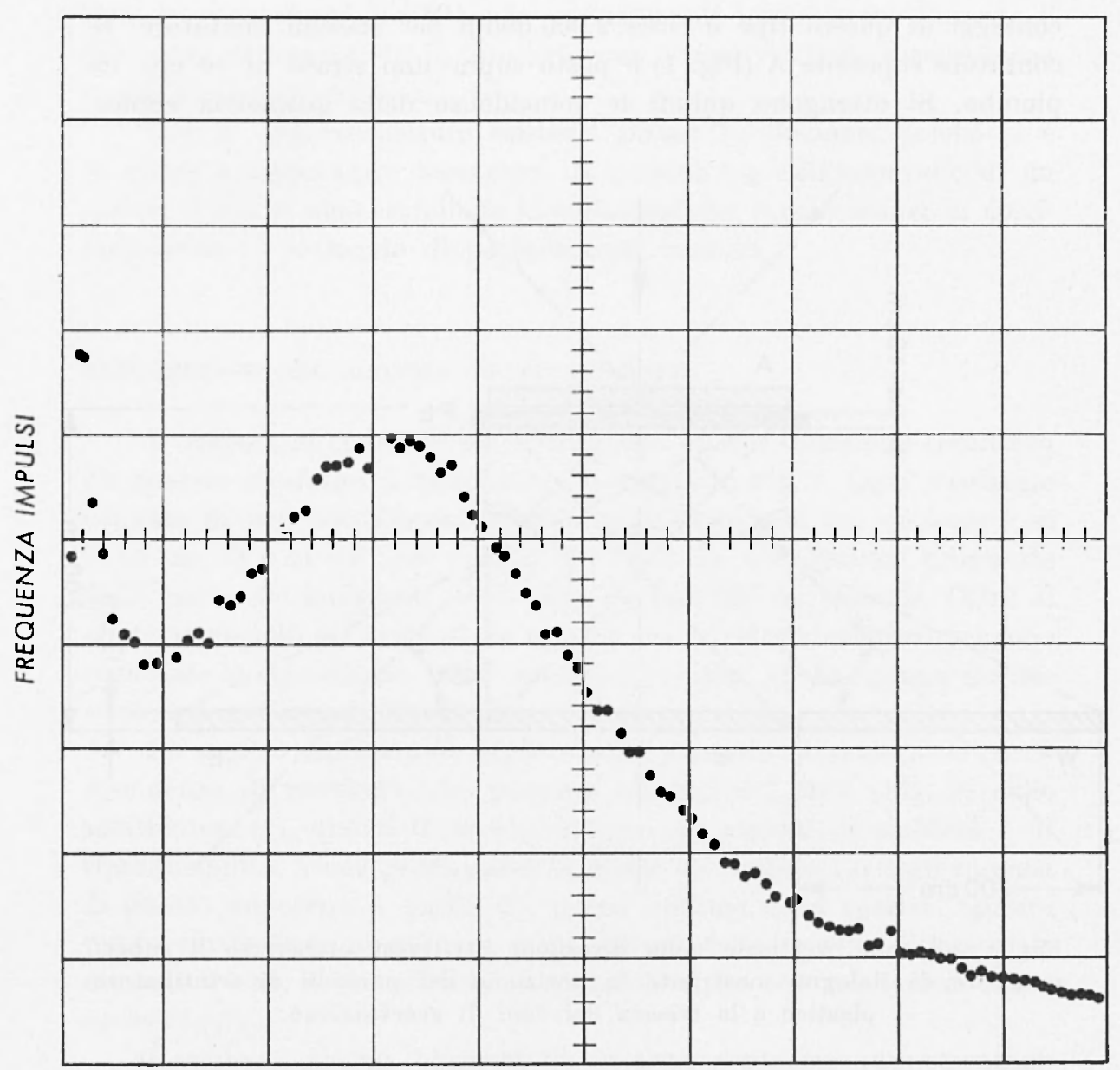

ALTEZZA IMPULSI

Fig. 2 - Spettro degli impulsi provenionti direttamente dai fototubi del supermonitore, ottenuto da un analizzatore a cento canali; le intensità e la frequenza degli impulsi sono in unita arbitrarie.

\section{OSSERVAZIONI FATTE.}

Si sono esaminati i dati della radiazione secondaria totale, ottenuti nella maniera sopradescritta, per gli anni 1965-66. Si sono poi considerate le variazioni di intensità in corrispondenza al passaggio di perturbazioni frontali a carattere freddo ed a carattere caldo. Al livello di 
sensibilità del supermonitore non si è potuto constatare che l'intensitì della totale presenti variazioni rapide al passaggro dei fronti caldi; ma si sono invece trovate variazioni caratteristiche in corrispondenza di fronti freddi. Queste variazioni, aventi una durata che non supera la decina di quarti d'ora ed una ampiezza non superiore all' $1,5 \%$ verranno chiamate microvariazioni. Dette microvariazioni si presentano (Fig. 3) come abbassamenti od inmalzamenti rapidi; od anche come un alternarsi di abbassamenti ed innalzamenti [caso (l), e) Fig. 3]. Spesso si verifica anche che, un abbassamento di notevole intensità è immediatamente preceduto e seguito da un imnalzamento [caso b), Fig. 3]. In generale gli abbassamenti prevalgono sugli innalzamenti. Comunque caratteristica comune di questi eventi è rhe il loro verificarsi lascia apparentemente inalterato l'andamento preesistente dell'intensilà. Queste microvariazioni compaiono sistematicamente al massimo due ore dopo il passagrgio di un fronte freddo al suolo od in quota; in generale sono contemporanee ad un temporale sopra la stazione. Infatti su un totale di 30 temporali registrati all'aeroporto di Borgo Panigale (distante circa $6 \mathrm{~km}$ dalla stazione di raggi (cosmici) nel 1966, circa 25 di essi sono stati accompagnati da una microvariazione (*).

Apparentemente in corrispondenza di pioggia abbondante l'evento di laggi cosmici è meno appariscente o addirittura assente. La microvariazione si mostra invece rilevante quando il temporale è di breve durata.

A titolo d'esempio nella Fig. 4 viene presentata una tipica microvariazione dei raggi cosmici corredata dai dati corrispondenti di temperatura nuvolosità pioggia e pressione al suolo e nelle Fig. $5 a$ e $5 b$ le relative carte sinotiche descriventi la situazione meteorologica del 23 Febbraio 1966.

Poiché durante i temporali dovuti al passaggio di fronti freddi è in giuoco l'elettricità atmosferica ed allo stesso tempo si ha ragione di ritenere che avrengano anche brusche variazioni di temperatura, si

$\left(^{*}\right)$ Questo è il numero di temporali pervenutoci dalle registrazioni fatte al Posto d'Informazione Meteorologica di Borgo Panigale della Direzione delle Tolecomunicazioni, lit Reg. Aerea di Milano, nell'anno 1966. Detto numero rappresenta la quasi totalita dei temporali avvenuti durante il 1966 su Borgo Panigale; vi sono infatti esclusi soltanto i temporali avvenuti dalle h 18.000 alle h 03.00 , periodo di tempo durante il quale il Posto di Informazione Meteorologica di Borgo Panigale non effettua registrazioni. 
a)

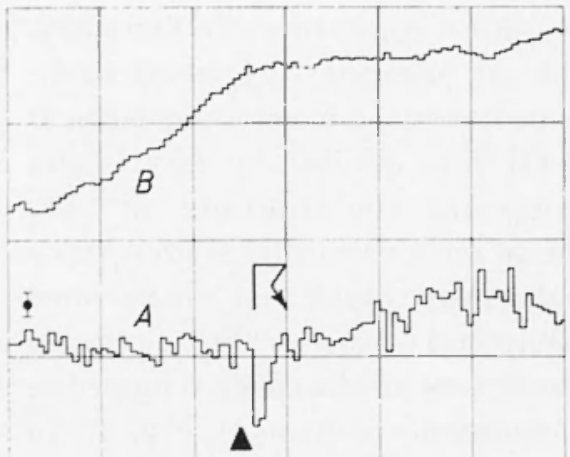

16

$$
\text { 27.28 Maggio } 1965
$$
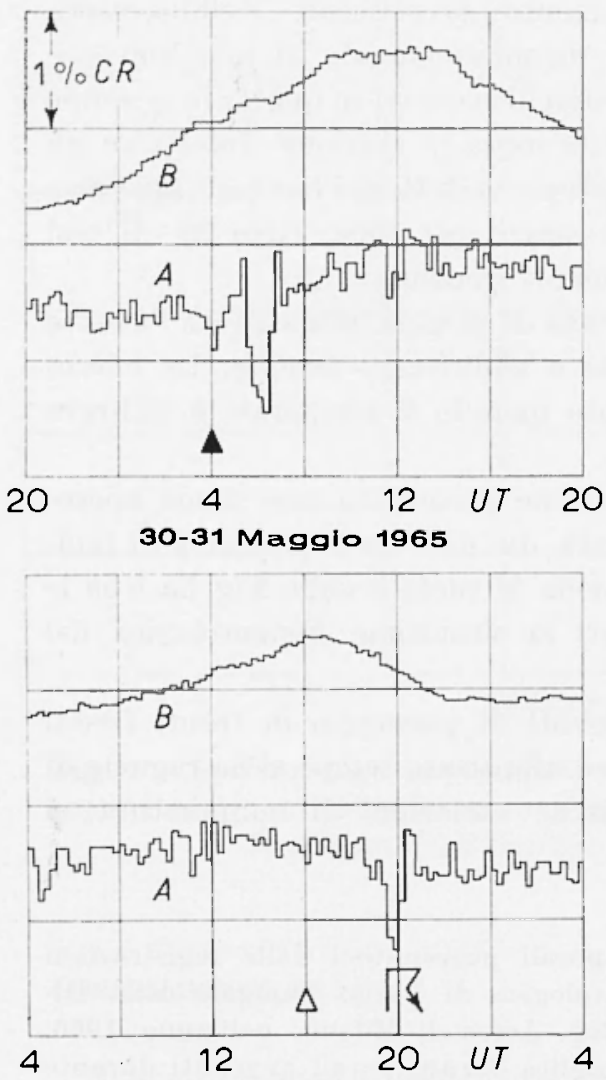

5 Giugno 1965
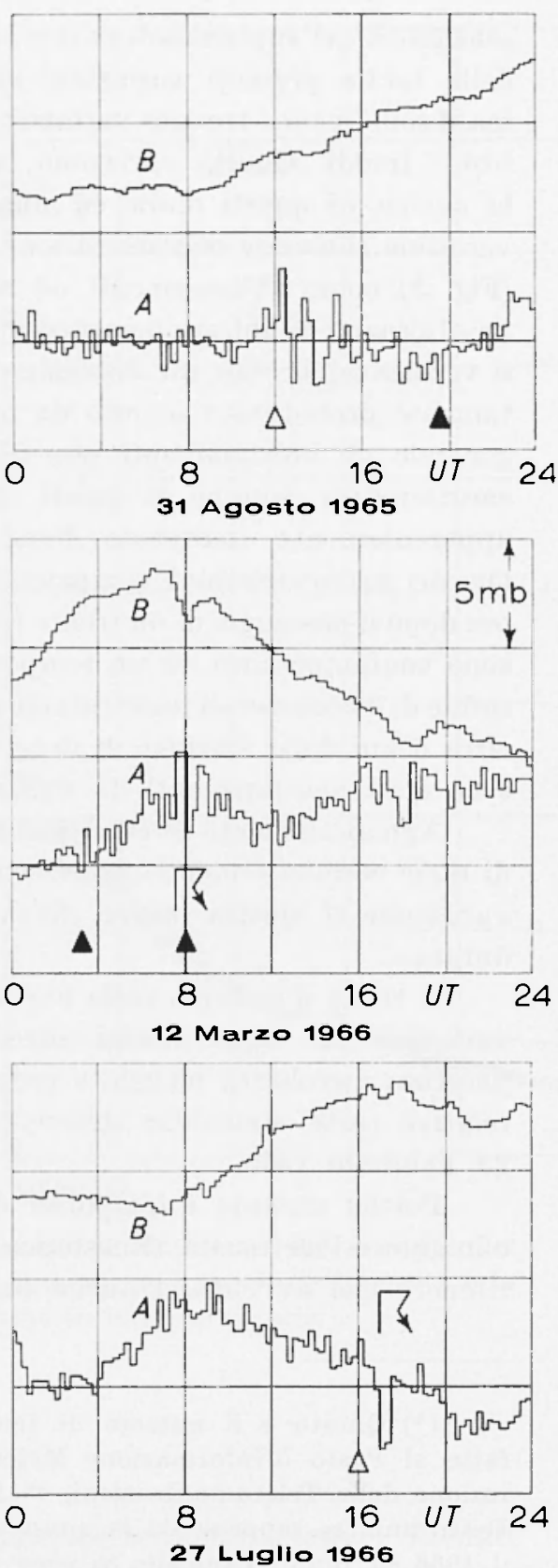

e)

d)

Fig. 3 - Alcuni esempi di microvariazioni di origine atmosferica; notare in $d$ ), e) l'alternarsi delle oscillazioni, ed in b) gli innalzamenti che precedono e seguono l'abbassamento; la scala delle pressioni è invertita. Curva $A \Rightarrow$ raggi cosmici - Curva $B=$ pressione. 


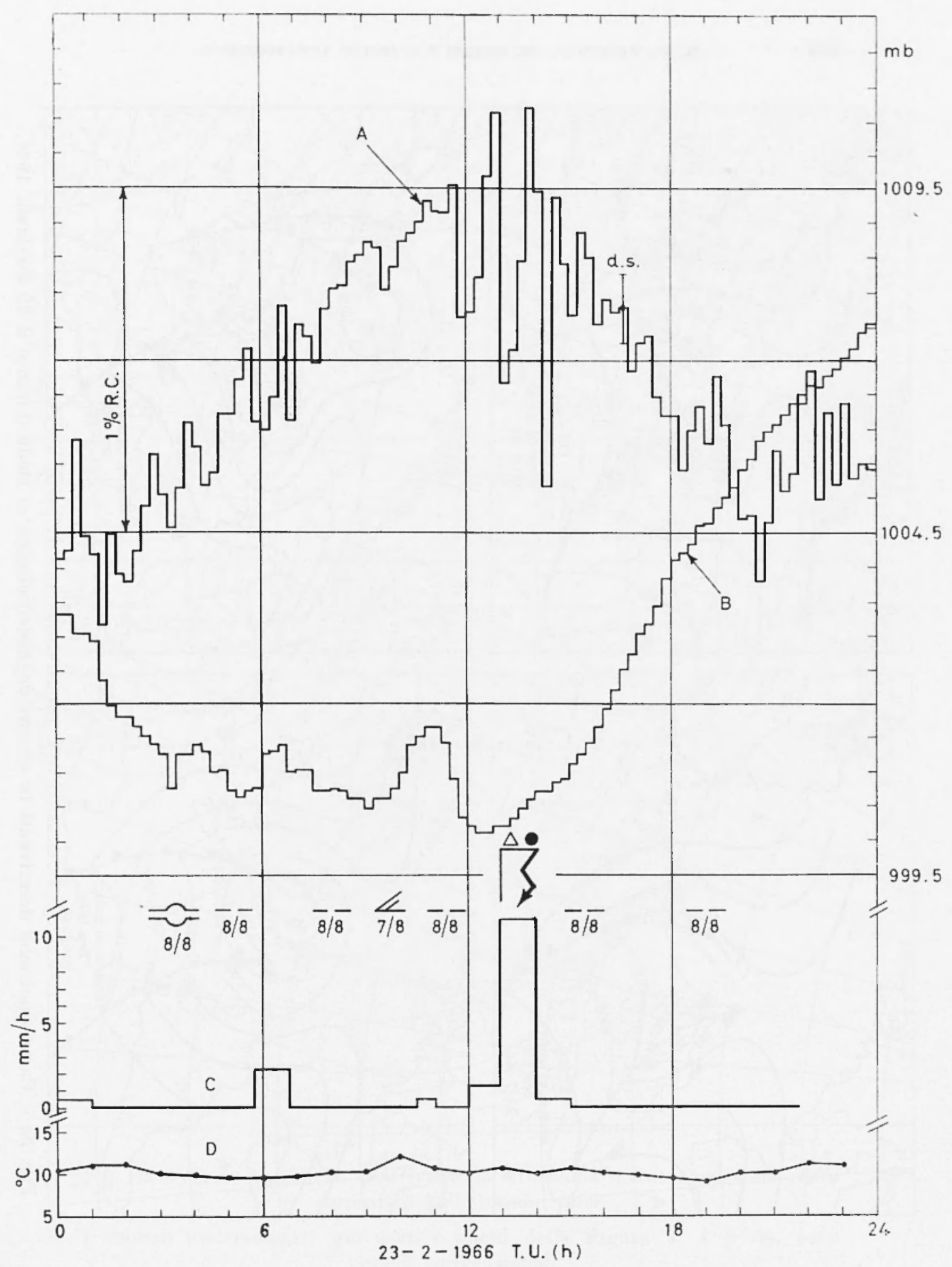

Fig. 4 - Evento del 23 Febbraio 1966 con le indicazioni della pressione, temperatura, nuvolosità, pioggia.

Curva $A=$ raggi cosmici - Curva $B=$ pressione

Curva $\mathrm{C}=$ pioggia - (urva $\mathrm{D}=$ temperatura. 

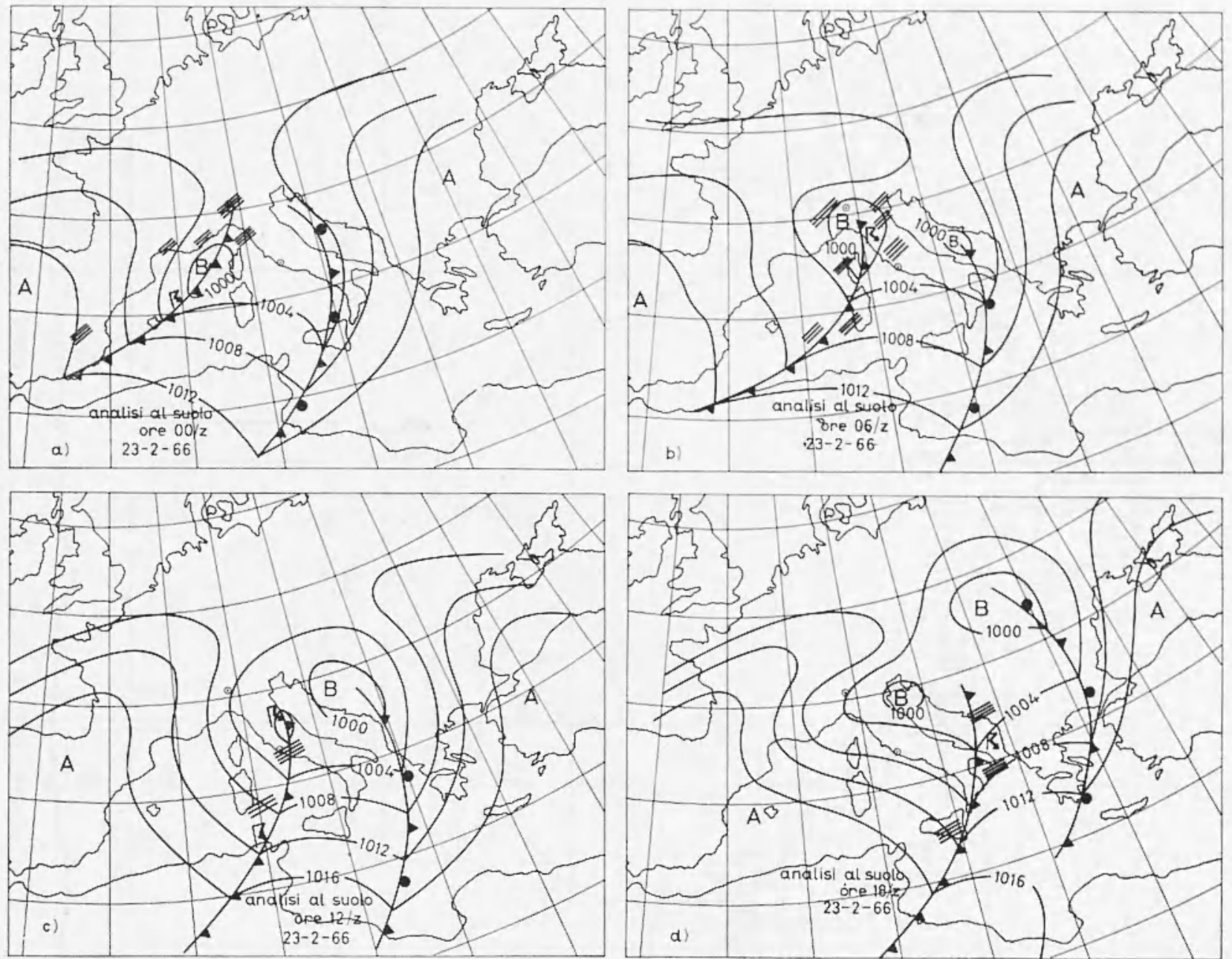

Fig. 5a - Carte sinottiche descriventi la situazione ${ }^{\top m}$ eteorologica al suolo durante il 23 Febbraio 1966. 

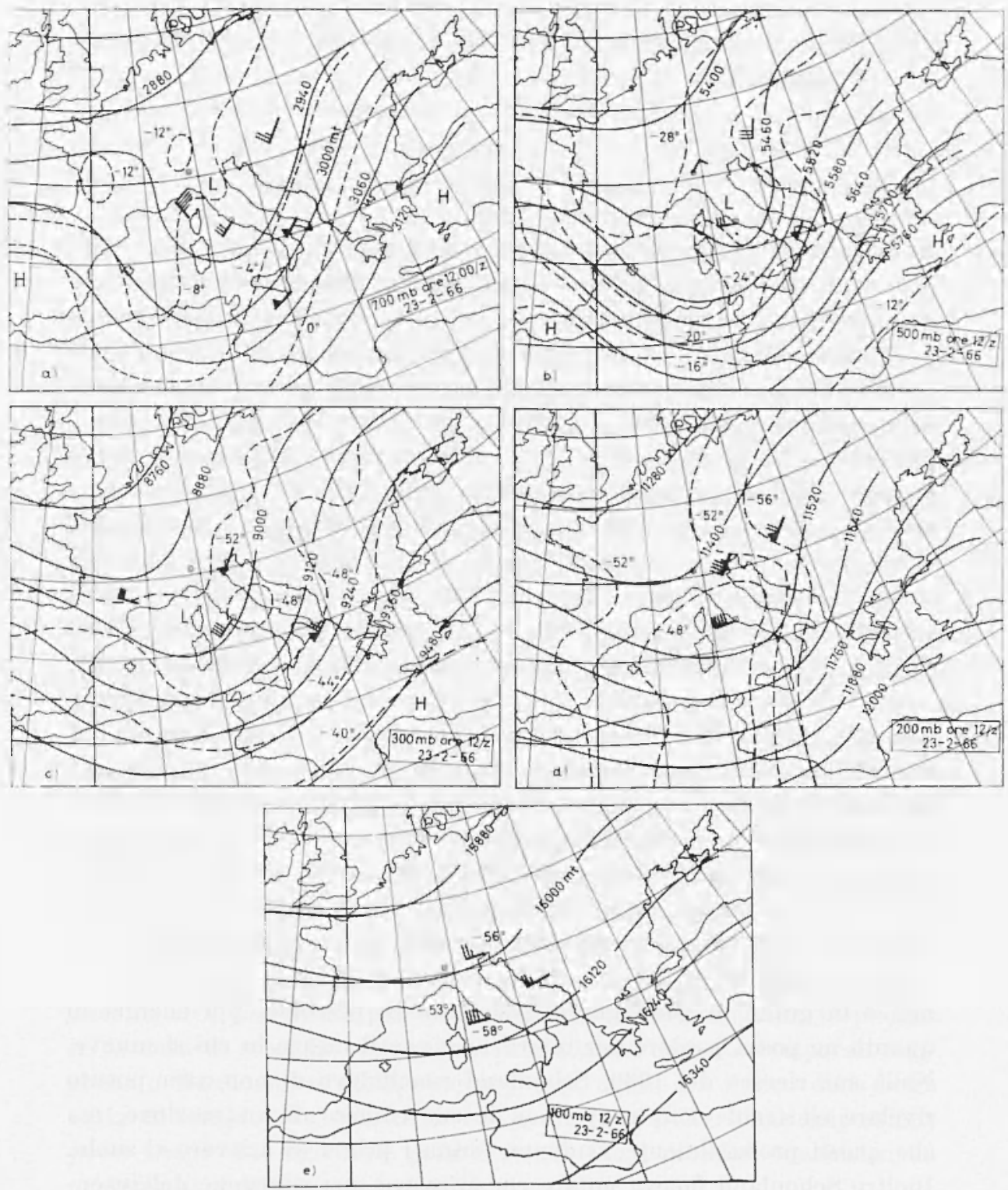

Fig. $5 b$ - Carte sinottiche descriventi la situazione meteorologica in quota durante il 23 Febbraio 1966.

I simboli metereologici qui usati e quelli delle Figure 3,4 e $5 a$, sono quelli conven\%ionali. 
ritiene che l'elettricità atmosferica e la temperatura, possano essere le uniche cause da indagare per spiegare le microvariazioni di raggi cosmici sopra descritte.

Possibili EFFETTI DEL CAMPo ELETTRICo ATMOSFERICO.

Agli inizi degli studi sui raggi cosmici, quando ancora non se nə conosceva né l'origine né la causa, l'effetto dell'elettricità sui raggi cosmici ha attirato l'attenzione dei maggiori studiosi di quei tempi.

Così C.T. R. Wilson $\left({ }^{10}\right)$, che già si era occupato dell'elettricità atmosferica - ed i raggi cosmici sono stati scoperti attraverso lo studio della elettricità atmosferica poiché si riteneva che avessero origine da elettroni amaloghi ai $\beta$ della radioattività - pensò che gli elettroni delle sostanze radioattive contenute nell'aria potessero venire accelerati durante i temporali. Elettroni di questo tipo, da lui chiamati elettroni " run-away " sono stati oggetto di ricerca fino ai nostri giomi ( $\left.{ }^{11}\right)$ C. T. R. Wilson reputava che una nube temporalesca, entro la quale supponeva esistessero differenze di potenziale dell'ordine di alcuni miliardi di Volt, potesse sparpagliare verso l'alto una corrente di elettroni. Egli diceva che questi elettroni “avrebbero acquistato una velocità tale da cvitare urti con le molecole d'aria ", e perciò li chiamò elettroni "run-away "; questi poi avrebbero dovuto ricadere a qualche distanza dalla nube in quanto deflessi dal campo magnetico terrestre.

Gli elettroni "run-away " sono stati cercati da Schonland (1930) (1) e da Schonland e Wiljoen (1933) $\left({ }^{2}\right)$. Altri Autori $\left({ }^{10},{ }^{11}\right)$ hamno fatto l'ipotesi che potessero avere origine dalla regione ionizzata della scarica temporalesea a causa dell'alta temperatura, della bassa densità e dei campi elettrici coinvolti. Infatti un elettrone non può "run-away " se non è in grado di guadagnare, per unità di percorso, più energia di quanta ne possa perdere per interazione con il mezzo in cui si muove. Nella sua ricerea del 1930, Schonland concludeva di non aver potuto rivelare elettroni "run-away" con le sue camere di ionizzazione, ma che questi probabilmente venivano fermati prima di arrivare al suolo. Inoltre Schonland faceva notare che si aveva una riduzione dell'intensità delle radiazioni quando la nube temporalesca era sovrastante. Si deve considerare che questi dati derivano da saggi di raggi cosmici presi saltuariamente durante giomate belle, scelte a caso, oppure in presenza di temporali ed a varie distamze da essi, senza però tener conto esplicitamente delle variazioni sia dell'efficienza dell'apparecehio da 
una volta all'altra sia dell'effetto di pressione o degli effetti primari. Schonland trovava variazioni nell'intensità comprese tra il $12 \%$ e il $39 \%$, rispetto ai valori durante il tempo bello; ma poichè nella letteratura successiva variazioni di simile entità, benché molto cospicue, non sono più state fatte rilevare, non sembra possibile trarre deduzioni dai suoi dati.

Schonland, constatando che la diminuzione el'a maggiore per quelle nubi in cui il campo risultava essere diretto verso l'alto, concludeva che la radiazione cosmica primaria dovesse essere formata in buona parte anche da particelle positive. In un successivo articolo del 1933, l'Autore facendo coincidenze con contatori Geiger tra lampi ed impulsi di particelle (la coincidenza con i fulmini viene ricercata come indice della preesistenza di forti campi elettrici), attribuisce le coincidenze sistematiche tra impulsi e lampi, ed il numero maggiore di impulsi appena prima del lampo, ad un effetto di radiazioni emesse dalla nube temporalesca. Egli non trovó però nessun effet to per temporali distanti meno di $30 \mathrm{~km}$ dal contatore.

Nel $193 \pm$ Halliday $\left(^{3}\right)$, con espansioni di camere a nebbia comandate da lampi di temporali distanti tra 0 e $40 \mathrm{~km}$, trovava che la probabilità di rivelare almeno una traccia in più in corrispondenza di lampi non er'a superiore al $10 \%$.

In tutti questi lavori è sempre tenuta presente l'ipotesi che le nubi temporalesche potessero contribuire alla accelerazione delle particelle con una differenza di potenziale dell'ordine di alcuni GV. Mentre le esperienze di Schonland riguardano le osservazioni di intensità in intervalli di alcuni secondi dalle seariche elettriche durante un temporale, Clay (1952) (1) riporta osservazioni fatte, su scala di tempo di alcune ore, durante temporali. Egli trova sempre aumenti (fino al $6 \%$ a seconda della schermatura della camera di ionizzazione e dei contatori) e riferisce di altri casi in cui furono osservate diminuzioni con forte pioggia in assenza di scariche elettriche e diminuzioni durante temporali registrati a Bandoeng e sul Hafelekar $\left({ }^{12}\right)$. A causa fra l'altro della larga scala di tempo coinvolta nelle sue osservazioni, si ritiene impossibile poter trarre, dal materiale pubblicato da Clay, ulteriori conclusioni in aggiunta a quelle da lui dedotte.

In realtà ad un esame qualitativo dei fatti sopra esposti risulta che non sono da escludersi effetti di accelerazione o decelerazione prorocati dai campi elettrici che accompagnano i temporali. Infatti Wilson $\left({ }^{10}\right)$ ha trovato che in un temporale le differenze di potenziale tra nube a nube o tra nube e terra, sono dell'ordine di $10^{\circ}$ Volt. Si possono 
avere differenze di potenziale nel senso sia da accelerare che da decelerare una particella secondaria di raggi cosmici; ovviamente la regione entro la quali tali differenze di potenziale possono esistere sarà confrontabile con le dimensioni della nube. E facile vedere che, tenuto conto dell'eccesso positivo dei $\mu$ e del taglio dei contatori (7 MeV), una differenza di potenziale di $0,2 \mathrm{GV}$ fra la nube, positiva o negativa, ed il suolo, sarebbe sufficiente per spiegare una variazione in eccesso od in difetto di circa l'1\% dell'intensità al suolo. E in corso attualmente uno studio approfondito sul reale effetto che l'attività elettrica può produrre sui raggi cosmici registrati in questa stazione.

\section{POSSIBILI GIFE'T'II DI TIEMPEIRATURA.}

In seguito alla scoperta del mesone $\mu$ e della sua instabilità (il mesome $\mu$, attualmente chiamato muone, veniva ritenuto la particella responsabile delle interazioni nucleari) vari Autori $\left({ }^{5}, 6\right)$ cercarono i possibili effetti del passaggio di fronti caldi e freddi sui raggi cosmici al suolo. Essi, confrontando le medie orarie dell'intensità col passaggrio di masse d'aria frontali, dopo aver fatto la correzione per la pressione atmosferica, trovarono effettivamente aumenti dell'intensità in occasione del passaggio di fronti freddi e diminuzioni al passaggio di fronti caldi; ovviamente la spiegazione era che ad un aumento di temperatura si doveva avere un immalzamento del livello di produzione dei $\mu$ e di conseguenza una diminuzione dell'intensità dei $\mu$ al suolo.

L'effetto di temperatura sui raggi cosmici fu poi studiato da un punto di vista più generale $\left({ }^{13}\right)$, soprattutto per quanto riguarda la temperatura del livello di produzione dei $\mu$. In una trattazione più completa, Dorman (1957) $\left({ }^{14}\right)$ ha correlato le variazioni di laggi cosmici con la distribuzione della temperatura nell'intero spessore dell'atmosfera e non con la temperatura di un particolare livello. In seguito a questa formulazione dell'effetto di temperatura, si introduce una quantità $W$ defnita come:

$$
W=\int_{o}^{x_{o}} W_{T}(x) \delta T(x) d x,
$$

dove $\delta T(x)$ - variazione di temperatura alla profondità atmosferica $x, W_{T}(x)=$ densità del coefficiente di temperatura, cioè una funzione che descrive il contributo dei vari livelli di aria alla produ- 
zione dell'efletto di temperatura alla profondità $x_{0}$. Perciò una qualsiasi variazione $\delta I$ dell'intensità $I$ di una certa componente alla profondità atmosferica $x_{0}$ si può esprimere con una equazione del tipo:

$$
\delta I=C_{N} \delta N+C_{P} \delta P+C_{W} \delta W
$$

dove $C_{N}, C_{P}$ sono coefficienti di regressione rispettivamente riferentesi ad una raliazione primaria $\delta N$, di pressione $\delta P$, e dove $W$ è il parametro di temperatura di Dorman.

E molto importante ai fini di questo studio, per quanto si vedrì tra poco, la forma della funzione $W_{T}(x)$. Detta funzione, calcolata da Ioorman, è rappresentata in Fig. 6; quelle trovate da Maeda e Wada $(19 \breve{5})\left({ }^{15}\right)$ e da Wada (1960) $\left({ }^{16}\right)$ hamno forma non molto diversa. Sicecome le variazioni per effetto di temperatura, corrispondenti a perturbazioni frontali considerate da Nishimura, Loughbridge e Dorman, sono su scala di tempo molto superiore alla scala di tempo delle microvariazioni qui trattate, a priori non si possono ritenere derivanti dalle stesse cause. Ciononostante un effetto di temperatura non si può escludere per queste microvariazioni in quanto condensazioni di vapore e successiva formazione di nubi provocano certamente aumenti locali e rapidi di temperatura di parecechi gradi $\left({ }^{17}\right)$. Questa interpretazione delle microvariazioni porterebbe a postulare l'esistenza di una "isola calda " nella sezione trasversile del fronte. Le oscillazioni multiple di intensit i potrebbero allora essere imputabili a spessori variabili delle nubi, o isole calde, sovrastanti i contatori; la variabilità negrli spessori potrebbe essere dovuta alla vorticosità dell'aria durante lo sviluppo di calore ed all'avanzare del fronte. Altrimenti sarebbe difficile rendere conto dello apparente ritorno rapido dell'intensita ai valori antecedenti la variazione.

Se la temperatura è la causa vera delle microviariazioni e la forma della densita del coefficiente di temperatura è circa costante con la profondità atmosferica (come indicato da Dorman $\left({ }^{14}\right)$, Maeda $\left({ }^{15}\right)$, Wada $\left({ }^{16}\right)$, e verificato da Lindstrom e Lindgren $\left({ }^{18}\right)$ e da Beroovitch $\left({ }^{19}\right)$ ), allora si può dire che le variazioni di ragresi cosmici risultano direttamente proporzionali alle variazioni del contenuto termico della colomna d'aria sovrastante i contatori.

Infatti si può pensare applicabile ai dati della totale omnidirezionale i risultati ottenuti per il telescopio cubico o semicubico da Dorman $\left(^{20}\right)$, tenuto conto che la frazione elettrofotonica che entra nel totale dei conteggi si puó stimare all'incirca il 10\%. Poiché il calore specifico dell'aria è $0,22 \mathrm{Cal} / \mathrm{kg}^{\circ} \mathrm{C}$ e poiché il valore medio di $W_{T}(h)$ è circa $-0,28 \%{ }^{\circ} \mathrm{C}^{-1} \mathrm{~atm}^{-1}$ (Fig. 6 ), si vede che la variazione di 1 oC su 
tutla la colonna d'aria di un $\mathrm{cm}^{2}$ di sezione, corrisponde ad un aumento della tolale ionizzante al suolo di $0,28 \%$. In altre parole, detta componento lotale dei ragrei cosmici diminuirì dell'1,3\% quando il contenuto

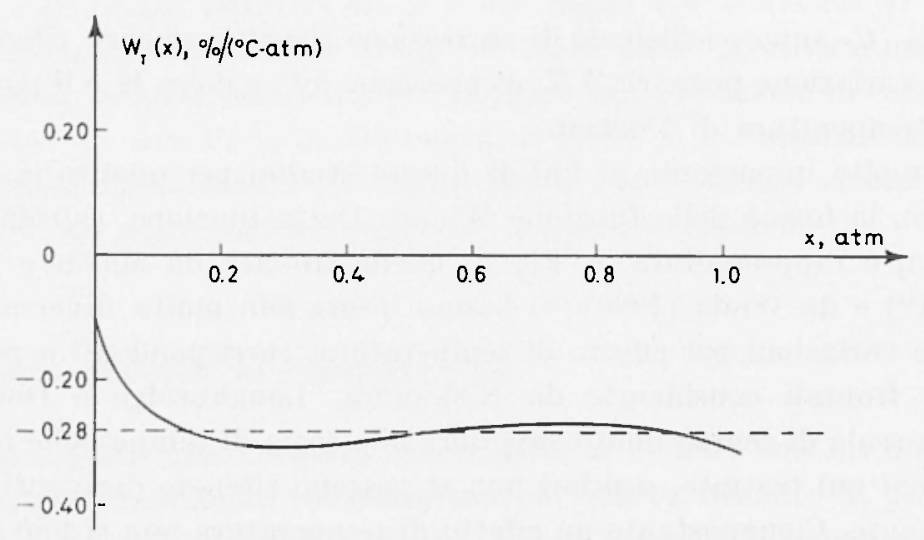

Fig. 6 - Andamento della densità del coefficiente di temperatura calcolato da L. I. Dorman per un telescopio cubico al livello del mare (curva a tratto intero); la curva tratteggiata indica il valore qui adottato.

termico dell'intera colonna d'aria sovrastante i contatori, aumenta di $1 \mathrm{Cal} / \mathrm{cm}^{2}$, corrispondente ad un aumento medio di temperatura di $4,5^{\circ} \mathrm{C}$, aumento compatibile con quello che avviene nel processo di formazione di nubi al passaggio di un fronte freddo.

\section{Conclusioni.}

Da quanto detto, risulta che ambedue le cause considerate per le microvariazioni, allo stato attuale delle conoscenze a della presente ricerea, potrebbero rendere ragione dell'entità delle variazioni rilevate. Si stanno raceogliendo ulteriori dati per ottenere maggiori risultati quantitativi tali da permettere di decidere fra le due alternative.

ì da notare che tra gli effetti clettrici, a priori non è da ascludere la possibilità di un effetto di "deflessione " da parte del campo elettrico atmosferico sulle particelle dei raggi cosmici, in modo da provocare variazioni di intensità al di sotto della nube temporalesca. 


\section{RINGRAZIAMENTI.}

Si ringraziano i Proff.: E. Rosini, O. Vittori e il Cap. I. De Barbieri, per le ptili discussioni sull'argomento; l'Ing. A. Gandolfi e il tecnico Sig. C. Merli per la loro ralida collaborazione nella riduzione ed elaborazione dei dati; il Cap. P. Rizzopenna ed il Posto d'Informazione Meteorologica di Borgo Panigale per le informazioni gentilmente fornite.

\section{BIBLIOGRAFIA}

(') Schonland B. F. J., Proc. Roy. Soc., A 130, 37 (1930).

$\left({ }^{2}\right)$ Scilonland B. F. J., Wilijoex J. P. T., Proc. Roy. Soc., A 140, 814 (1933).

( ${ }^{3}$ Halliday E. C., Proc. Camb. Soc., 30, 206 (1934).

(1) Clay J., Physica, 18, 801 (1952): Proc. Kon. Ned. Akad. IVel., 55 , $300 ; 450$ (1952).

(5) Nisilna Y., Arakawa H., Sekido Y., Simamura H., Phys. Rev., 57, $1050(1940)$.

(') Loughiridge D. Il., Gast P., Phys. Rev., 56, 1169 (1939).

(7) Rossi B., Rev. Mod. Phys., 20, 543 (1948).

(8) Allkofer O. C., Nuovo Cim., 39, 1055 (1965).

$\left({ }^{9}\right)$ Rossi B., High Energy Particles, Prentice-Hall Ine., New York, 1952, 337.

(10) Wilson C. T. R., Proc. Camb. Soc., 22, 534 (1925); Trans. Phil. Soc., London. 221, 73 (1920).

('1) Hilt R. D., J. Geophys. Res., 68, 6261 (1963).

(12) Steinmadrer R., Gerlands Beiträge. 45, 148 (1949).

(13) Duperier A., Proc. Phys. Soc., A 62, 684 (1949).

(14) Dorman L. I., Cosmic Ray Varialions, State Publishing House, Moscow, 1957 ,p. 170.

(15) MaEda K., Wada M., J. Sci. Res. Inst. Tokio, 48, 71 (1954).

${ }^{\left({ }^{16}\right)}$ Wada M., Sci. Paper Inst. Phys. Ohem. Res. Tokio, 54, 335 (1960).

(17) Borovikov A. M., Kurginy A. Kir, et al, Cloud Physics, Jerusalem, 1963 , p. 222.

(18) Lindgrex S., Lindiloly F., T'ellus, 13, 281 (1961).

(19) Bercovitch M., Carmicifale H., Steljes J. F., Tellus, 19, 143 (1967).

(20) Dorman L. I., Instruction of Meleorological Corrections to Data of Cosmic Ray Intensity, NIZMIR, Moscow, 1958.

(21) Petersen S., Weather Analysis and Forecasling, Me Graw-IIill Book Company Inc., New York, 1956. 